\title{
CURRENT OUTPUT FILTER FOR STATE ESTIMATION OF SCALAR NONLINEAR SYSTEMS WITH APPLICATION TO CHAOTIC COMMUNICATION
}

\author{
Huawei Ruan, Edwin Yaz and Tongyan Zhai \\ Department of Electrical and Computer Engineering \\ Marquette University \\ Milwaukee, WI 53201, USA
}

\begin{abstract}
A novel nonlinear state estimation technique called Current Output Filter is proposed in this paper. By taking two successive measurements into account and optimizing the coefficients of the proposed filter structure, an unbiased minimum variance state estimation algorithm with guaranteed estimation error performance is derived for scalar nonlinear systems. The performance of this filter is shown to be superior to the Extended Kalman Filter (EKF) in simulations. An application to a chaotic communication scheme is also presented in this work. Copyright ( 2005 IFAC
\end{abstract}

Keywords: state estimation, filter, nonlinear, chaos, communication

\section{INTRODUCTION}

The main purpose of this work is the introduction of a novel nonlinear state estimator design for onedimensional nonlinear systems. Due to the fact that there is always noise interference with the measurement of the states of a nonlinear system, state estimation techniques were developed to reduce the noise effect in order to retrieve more accurate information about the system states. A classical state estimation technique for nonlinear stochastic system is Extended Kalman Filter (EKF) (Lewis, 1986), which is the extension of Kalman Filter for linear systems and was designed based on a local linearization around the current state estimate. The estimation performance and convergence conditions for EKF have been investigated by many researchers, e.g. (Reif, Gunther, and Yaz etc., 1999;, Zhai, Ruan, and Yaz, 2003; Leung and Zhu, 2001). Although EKF is a well defined recursive locally optimal estimator for systems with differentiable nonlinearities, its performance may not always be desirable. We will consider a class of onedimensional nonlinear system and measurement models for our state estimator design. Such systems especially those that can exhibit chaotic behavior, are used a lot in some applications, for example, chaotic communication systems that benefit the broadband spectrum and noise like state trajectory of the signals generated from chaotic systems (Kennedy, Rovatti, and Setti, 2000; Kolumban, Kennedy and Chua, 1998). In these applications, EKF sometimes can not work effectively as a state estimator to reduce the effect of noise.

In this work, we develop a new state estimation technique, namely, Current Output Filter (COF), which can effectively reduce the noise effect for the state estimation of one-dimensional nonlinear systems. Instead of just using one previous measurement recursively for estimating system state in EKF, COF developed in this work uses both previous and current measurements (Yaz, 1992). Performance analysis and simulation results in this work show that this novel state estimator can effectively reduce the noise effect thus giving us an alternative demodulation approach for chaotic communication.

This paper is organized as follows: Section 2 introduces the design of the proposed filter and the performance analysis as well as its comparison with 
Extended Kalman Filter. Section 3 includes simulation studies of COF performance. Section 4 presents a possible application to a chaotic communication scheme and the performance comparison with EKF by simulation. The conclusions are drawn in section 5 .

\section{CURRENT OUTPUT FILTER}

Consider a discrete time system model that has state space representation,

$$
\begin{aligned}
& x_{k+1}=f\left(x_{k}\right) \\
& y_{k}=x_{k}+w_{k}
\end{aligned}
$$

where $x_{k}$ is a scalar state variable. $y_{k}$ is the output measurement and $w_{k}$ is white Gaussian noise with zero mean and $\sigma_{w}^{2}$ variance. In some recursive estimators, we only use one output measurement. In this current output filter, we use two output measurements for estimating the state variable of the system. The proposed structure of state estimation equation is:

$$
\hat{x}_{k+1}=K_{1}\left(\hat{x}_{k}\right)+K_{2} y_{k}+K_{3} y_{k+1}
$$

The following summarizes our main results:

Theorem: Let filter (2) be used in estimating the state of system (1) from its noisy measurements. The first order approximation to the unbiased minimum variance filter is given by the following choice of coefficients:

$$
\begin{aligned}
& \hat{x}_{k+1}=\frac{1}{1+A_{k}^{2}} f\left(\hat{x}_{k}\right)+\frac{A_{k}}{1+A_{k}^{2}}\left(y_{k}-\hat{x}_{k}\right) \\
& +\frac{A_{k}^{2}}{1+A_{k}^{2}} y_{k+1}
\end{aligned}
$$

where $A_{k}=\left.\frac{\partial f}{\partial x}\right|_{x=\hat{x}_{k}}$, and the mean square error is given by

$$
E\left\{e_{k}^{2}\right\}=\frac{A_{k-1}^{2}}{1+A_{k-1}^{2}} \sigma_{w}^{2}<\sigma_{w}^{2}
$$

where $E\{\bullet\}$ denotes expectation.

The structure of this filter obviously has less computational complexity than EKF since it only involves the calculation of the first order derivative instead of both derivative and estimation error covariance in EKF. Therefore, COF can be updated much faster than EKF. Another obvious advantage of the filter is the availability of an exact expression for the mean square error at all times, which is a major improvement on the previous results [2] and [3] regarding the convergence of EKF.

\section{Proof:}

The estimation error has the following dynamics:

$e_{k+1}=x_{k+1}-\hat{x}_{k+1}$

$=f\left(x_{k}\right)-K_{1}\left(\hat{x}_{k}\right)-K_{2} y_{k}-K_{3} y_{k+1}$

$=f\left(x_{k}\right)-K_{1}\left(\hat{x}_{k}\right)-K_{2}\left(x_{k}+w_{k}\right)-$
Expanding $f\left(x_{k}\right)$ into Taylor series at $\hat{x}_{k}$,

$f\left(x_{k}\right)=f\left(\hat{x}_{k}\right)+\left.\frac{\partial f(x)}{\partial x}\right|_{x=\hat{x}_{k}}\left(x_{k}-\hat{x}_{k}\right)+\ldots$

We assume that the second and higher order of derivatives are negligible and obtain

$$
\begin{aligned}
& e_{k+1}=f\left(\hat{x}_{k}\right)+A_{k} e_{k}-K_{1}\left(\hat{x}_{k}\right)-K_{2}\left(\hat{x}_{k}+e_{k}+w_{k}\right) \\
& -K_{3}\left(f\left(\hat{x}_{k}\right)+A_{k} e_{k}+w_{k+1}\right) \\
& =\left(1-K_{3}\right) f\left(\hat{x}_{k}\right)-K_{2} \hat{x}_{k}-K_{1}\left(\hat{x}_{k}\right)+ \\
& \left(A_{k}-K_{2}-K_{3} A_{k}\right) e_{k}-K_{2} w_{k}-K_{3} w_{k+1} \\
& \text { ( } 6) \\
& \text { Letting } \\
& K_{1}\left(\hat{x}_{k}\right)=\left(1-K_{3}\right) f\left(\hat{x}_{k}\right)-K_{2} \hat{x}_{k} \\
& K_{2}=\left(1-K_{3}\right) A_{k}
\end{aligned}
$$

Thus, the expected value of the estimation error will be

$$
\begin{aligned}
& E\left\{e_{k+1}\right\}=E\left\{-K_{2} w_{k}-K_{3} w_{k+1}\right\} \\
& =-K_{2} E\left\{w_{k}\right\}-K_{3} E\left\{w_{k+1}\right\} \\
& =0
\end{aligned}
$$

So, we achieve unbiased estimation.

The mean square error of the estimator will be

$$
\begin{aligned}
& E\left\{e_{k+1}^{2}\right\}=E\left\{\left(-K_{2} w_{k}-K_{3} w_{k+1}\right)^{2}\right\} \\
& =E\left\{\left(K_{2} w_{k}\right)^{2}+\left(K_{3} w_{k+1}\right)^{2}-2 K_{2} K_{3} w_{k} w_{k+1}\right\} \\
& =K_{2}^{2} E\left\{w_{k}^{2}\right\}+K_{3}^{2} E\left\{w_{k+1}^{2}\right\}-2 K_{2} K_{3} E\left\{w_{k} w_{k+1}\right\} \\
& =\left[\left(1-K_{3}\right)^{2} A_{k}^{2}+K_{3}^{2}\right] \sigma_{w}^{2} \\
& =\left[\left(1+A_{k}^{2}\right) K_{3}^{2}-2 A_{k}^{2} K_{3}+A_{k}^{2}\right] \sigma_{w}^{2}
\end{aligned}
$$

When $K_{3}=\frac{A_{k}^{2}}{1+A_{k}^{2}}$, we get the minimum value of this quadratic function, which

is $E\left\{e_{k+1}^{2}\right\}=\frac{A_{k}^{2}}{1+A_{k}^{2}} \sigma_{w}^{2}$.

Accordingly, we get

$$
\begin{aligned}
& K_{2}=\left(1-K_{3}\right) A_{k}=\frac{A_{k}}{1+A_{k}^{2}} \\
& K_{1}\left(\hat{x}_{k}\right)=\left(1-K_{3}\right) f\left(\hat{x}_{k}\right)-K_{2} \hat{x}_{k} \\
& =\frac{1}{1+A_{k}^{2}} f\left(\hat{x}_{k}\right)-\frac{A_{k}}{1+A_{k}^{2}} \hat{x}_{k}
\end{aligned}
$$

Thus giving us equations (3) and (4). This concludes the proof.

Remark 1. Since $\frac{A_{k}^{2}}{1+A_{k}^{2}}<1$, the mean square error of estimation in (4) is less than $\sigma_{w}^{2}$.

Remark 2. By setting the coefficients of $E\left\{e_{k}\right\}$ to zero, it will not be necessary to know $E\left\{x_{0}\right\}$ to set $\hat{x}_{0}=E\left\{x_{0}\right\}$.

$K_{3}\left(f\left(x_{k}\right)+w_{k+1}\right)$ 
Remark 3. Obviously, if the second and higher order terms of the Taylor series are all zero, this filter will achieve the best performance. However, even if the second and higher order derivatives are non-zero, the filter can still work better than classical EKF-based state estimation.

\section{PERFORMANCE STUDY}

Figure 1 shows the mean square error performance of this filter for three different kinds of scalar chaotic dynamics, two of which have second degree polynomial nonlinearities. Current Output Filter has been applied to three different types of scalar nonlinear systems, tent map, quadratic map and logistic map, all of which are operating in their chaotic regimes.

The functions of the three maps we tried in the simulation is as follows:

Tent map: $x_{k+1}=2-1.6\left|x_{k}-1\right|$

Quadratic map: $x_{k+1}=x_{k}^{2}-2$

Logistic map: $x_{k+1}=4 x_{k}\left(1-x_{k}\right)$

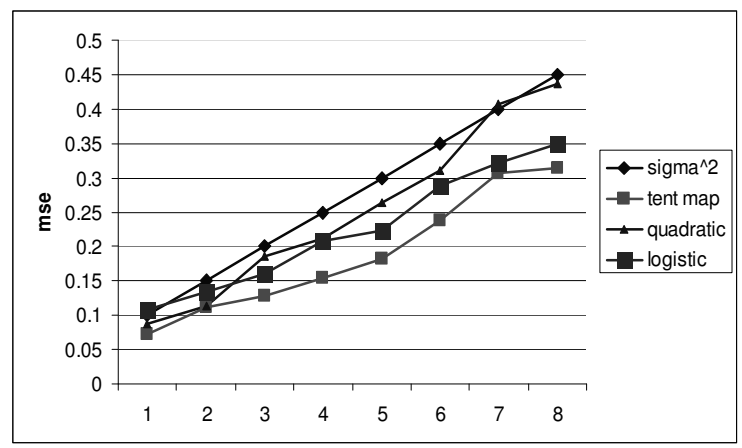

Figure 1: MSE of Current Output Filter

Figure 1 shows the relative mean square estimation errors for various maps as the measurement noise variance is increased. From the simulation shown in Figure 1, we can see that COF works the best for linear or piecewise linear systems due to the bigger linearization error for second order polynomial maps. Figure 2 is the mean square error performance of EKF for the same three maps under the same noise levels. One can clearly see by comparing Figures 1 and 2 that COF has much superior performance not only for first degree but also for second degree nonlinear functions.

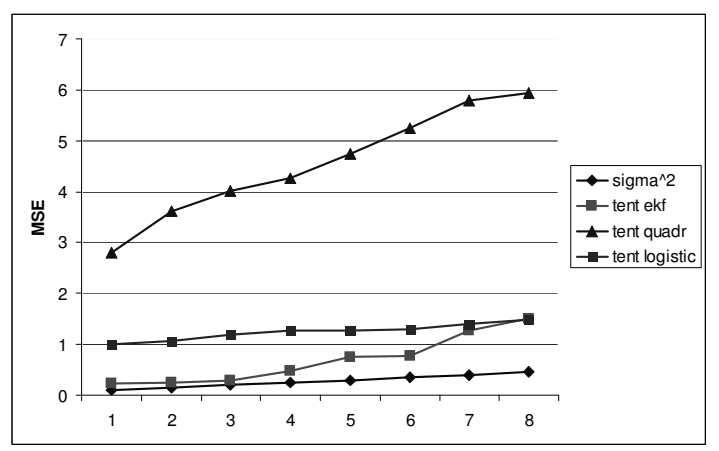

Figure 2: MSE of EKF for various maps

\section{APPLICATION TO CHAOTIC COMMUNICATION}

We propose an application of Current Output Filter to a chaotic communication scheme we have developed before in which EKF was employed as the demodulator at the receiver (Ruan, Zhai and Yaz, 2003).

The block diagram of the communication system is given in Figure 3:

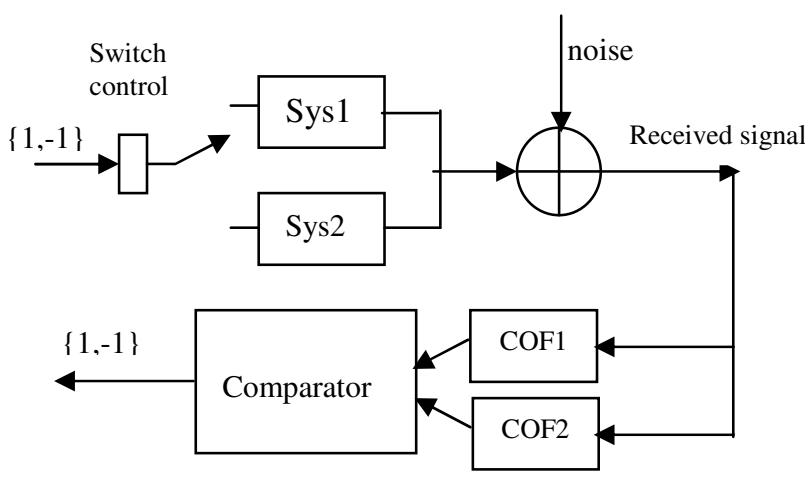

Figure 3: Proposed communication scheme

This communication scheme is designed to transmit digital messages by chaotic modulation/demodulation. The binary messages are represented by finite-length chaotic sequences generated by scalar nonlinear systems with different parameters. By modulating the digital message this way, we can get broadband and noise like signals transmitted through the channel thus increasing the security level and broader spectrum utilization. At the receiver, two COFs are built with respect to the two parameters of the nonlinear systems that represent the binary digital symbols. Due to the fact that parameter mismatch between the real system model and the one used in the estimator will cause large estimation error, the parameter associated with the received chaotic sequence that contains the digital message can be found by comparing the state estimation errors of the two COFs. Thus, the transmitted digital message can be recovered accordingly. We consider a one-dimensional chaotic map for modulation purposes:

$$
x_{k+1}=f\left(x_{k}, a\right)
$$

If we want to send 1 , we send out a chaotic sequence generated by the function with parameter $a_{1}$; if we want to send -1 , we send out a sequence generated by the function with parameter $a_{2}$. Suppose the length of each chaotic sequence is $L$ and the frequency of the digital signals is $f$, then the sampling frequency of the chaotic signals will be $L f$. For example, to transmit a single digital signal, an $L$ bit chaotic sequence with randomly generated initial condition is needed. So, the transmitted sequence for one bit will be $\left[x_{1}, x_{2}, \ldots, x_{L}\right]$. We only consider the AWGN channel. So, at the receiver, the measurement will be: 


$$
y_{k}=x_{k}+w_{k}
$$

where $x_{k}$ is the state of one of the two chaotic systems and $w_{k}$ is white Gaussian noise with zero mean and variance $\sigma^{2}$.

The receiver has the knowledge of the structure and possible parameters of the chaotic systems, so two Current Output Filters based on the two possible parameters of the chaotic system are built to estimate the state of these chaotic systems. As the estimate of $x_{k}$, the first COF with parameter $a_{1}$, we obtain the estimate $\hat{x}_{1 k}$ and similarly $\hat{x}_{2 k}$ is obtained from the second EKF with the assumed parameter $a_{2}$. The estimation error used to determine whether there is filter mismatch for each filter would be sample mean square errors:

$$
\begin{aligned}
& E_{1}=\frac{1}{L} \sum_{k=1}^{L}\left(y_{k}-\hat{x}_{1 k}\right)^{2} \\
& E_{2}=\frac{1}{L} \sum_{k=1}^{L}\left(y_{k}-\hat{x}_{2 k}\right)^{2}
\end{aligned}
$$

So, for sufficiently large L, the filter with the correct parameter match will give rise to an error much smaller than the other one. That is how we can decide whether the transmitted message is +1 (if $E_{1}<E_{2}$ ) or -1 (if $E_{1}>E_{2}$ ).

In the simulation, we have used skew tent map to generate the chaotic sequence for modulating the binary digital messages. The Skew Tent map is defined by

$$
x_{k+1}=\left\{\begin{array}{c}
x_{k} / a, \quad 0 \leq x_{k} \leq a \\
\left(1-x_{k}\right) /(1-a), \quad a<x_{k} \leq 1
\end{array}\right.
$$

The two values of parameter $a$ we have used in this simulation to generate the chaotic sequences are 0.3 and 0.7 respectively. The length of each sequence is 100 in this simulation. The Bit Error Rate performance of the proposed system under different noise level is studied by conducting Monto Carlo simulation. The noise level is described by Signal to Noise Ratio, which is defined as follows:

$$
\mathrm{SNR}=10 \log \frac{E_{b}}{E_{n}}(\mathrm{~dB})
$$

where $E_{b}$ and $E_{n}$ are signal and noise energies respectively.

The Bit Error Rate (BER) performance of this system with EKF as the demodulator is also shown in Figure 4. We can see that COF works much better than EKF in this chaotic communication scheme.

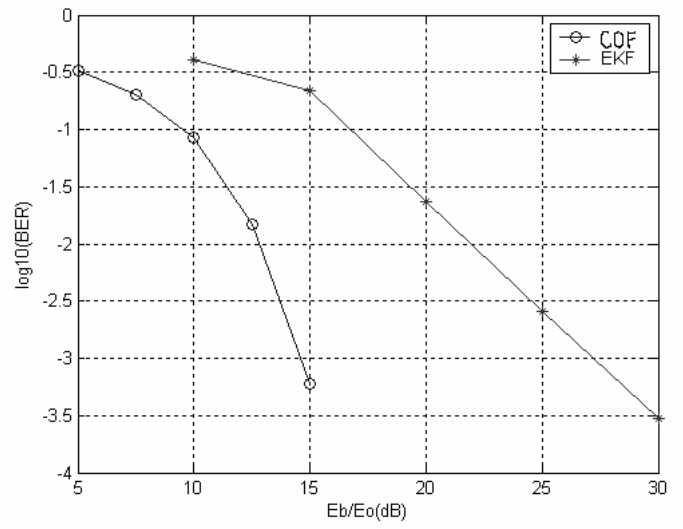

Figure 4: BER performance of proposed communication system

\section{CONCLUSION}

In this work, we have proposed a new state estimation algorithm for scalar nonlinear dynamic systems. By introducing two successive measurements and optimizing the filter structure, we have developed a state estimator that can effectively reduce the effect of noise for the state estimation of scalar nonlinear systems. The theoretical analysis and simulation studies show that this filter works much better than classical EKF. We have also successfully applied this filter to a chaotic communication scheme that was developed in (Ruan, Zhai and Yaz, 2003). with EKF based nonlinear estimation technique. The simulation results show that the proposed system achieves much better performance with COF than with EKF.

\section{REFERENCES}

Kennedy, M., Rovatti, R. and Setti, G. (2000).

Chaotic Electronics in Telecommunications, CRC Press, Boca Raton, FL, USA.

Kolumban, G., Kennedy, M. and Chua, L. (1998).

The Role of Synchronization in Digital Communications Using Chaos-Part II: Chaotic Modulation and Chaotic Synchronization, IEEE Trans. Circuits and Sys., vol. 45, pp. 1129-1139.

Leung, H. and Zhu, Z. (2001). Performance

Evaluation of EKF-based Chaotic Synchronization, IEEE Trans. Circuits and Systems-I: Fundamental Theory and Applications, vol. 48, pp. 1118-1125.

Lewis, F. L. (1986). Optimal Estimation, Wiley: New York.

Reif, K., Gunther, S., Yaz, E. and Unbehauen, R. (1999). Stochastic Stability of the EKF: the Discrete-Time Case, IEEE Trans. Autom. Contr., vol. 44, pp. 714-728.

Ruan, H., Zhai, T. and Yaz, E. (2003). A

Demodulation Scheme Based on State Estimation for Chaotic Digital Communication. Proceedings of 2003 American Control Conference, Denver, CO, pp. 1614-1618.

Yaz, E. (1992). Full and Reduced-Order Observer 
Designs for Discrete Stochastic Bilinear Systems, IEEE Trans. on Automatic Control, vol. 37, pp. 503-505.

Zhai, T., Ruan, H. and Yaz, E. (2003). Performance Evaluation of Extended Kalman Filter Based State Estimation for First Order Nonlinear Dynamic Systems, Proceedings of IEEE International Conference on Decision and Control, Hawaii, pp. 13861391. 